\title{
Semi-Interpenetrating Polymer Networks for Enhanced Supercapacitor Electrodes
}

Kara D. Fong, ${ }^{\dagger}$ Tiesheng Wang, ${ }^{\dagger}$ Hyun-Kyung Kim, R. Vasant Kumar and Stoyan K. Smoukov*

Department of Materials Science and Metallurgy, University of Cambridge,

27 Charles Babbage Rd., Cambridge CB3 0FS, United Kingdom

$\dagger$ These authors contributed equally to this work.

* Author to whom correspondence should be addressed, sks46@cam.ac.uk. 


\section{ABSTRACT}

Conducting polymers show great promise as supercapacitor materials due to their high theoretical specific capacitance, low cost, toughness, and flexibility. Poor ion mobility, however, can render active material more than a few tens of nanometers from the surface inaccessible for charge storage, limiting performance. Here, we use semi-interpenetrating networks (sIPNs) of a pseudocapacitive polymer in an ionically conductive polymer matrix to decrease ion diffusion length scales and make virtually all of the active material accessible for charge storage. Our freestanding poly(3,4-ethylenedioxythiophene)/poly(ethylene oxide) (PEDOT/PEO) sIPN films yield simultaneous improvements in three crucial elements of supercapacitor performance: specific capacitance (182 F/g, a 70\% increase over neat PEDOT), cycling stability (97.5\% capacitance retention after 3,000 cycles), and flexibility (the electrodes bend to $<200 \mu \mathrm{m}$ radius

of curvature without breaking). Our simple and controllable sIPN fabrication process presents a framework to develop a range of polymer-based interpenetrated materials for high-performance energy storage technologies.

\section{TOC GRAPHIC}

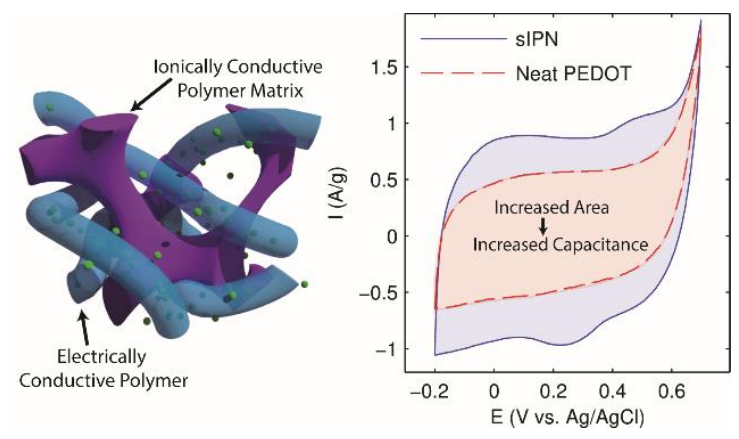


Supercapacitors present a promising means to meet the demand for improved energy storage technologies, combining the high energy density of batteries and the high power density of conventional capacitors. ${ }^{1}$ Their superior energy storage capability, long-term stability, and low maintenance requirements make them suitable for a variety of applications, such as regenerative braking and power supply in hybrid vehicles, memory backup for portable electronics, and power buffering or load-leveling for industrial scale systems. ${ }^{2,3}$ Today's commercially available supercapacitors, however, are typically bulky and rigid, limiting their implementation in many cutting-edge electronics. Much work in recent years has thus been devoted to develop lightweight, flexible supercapacitors to enable novel technologies such as wearable electronics, roll-up displays, or bio-implantable devices. ${ }^{4,5}$

Conducting polymers have emerged as competitive materials for this new class of supercapacitors based on their high energy and power densities, low cost, high conductivity, and robust mechanical properties such as flexibility and stretchability. ${ }^{6,7}$ However, poor ion mobility within conducting polymer electrodes often hinders the performance of these materials. At fast charging/discharging rates, active material which is not within the first few tens of nanometers of the electrode-electrolyte interface will be inaccessible to the electrolyte ions, rendering this material unutilized and lowering specific capacitance. ${ }^{8,9}$

Considerable research efforts have been devoted to addressing this ion mobility issue by forming polymer nanostructures or composite hierarchical structures which decrease ion diffusion lengths within the electrode. ${ }^{10}$ Specific capacitance can be drastically improved by depositing thin layers of conducting polymer onto high surface area supports, such as metal oxide nanowire arrays ${ }^{11,12}$ or carbon nanotube networks. ${ }^{13-15}$ These structures allow for high mass loadings without burying much of the active material too far from the electrode-electrolyte interface to participate in charge 
storage reactions. Hydrogels or other porous structures can also improve ion mobility by facilitating electrolyte infiltration into the electrode bulk. ${ }^{16-18}$

While these approaches have largely succeeded in contributing to supercapacitors with facile ion transport properties and high specific capacitance, many of these nanostructuring or compositing techniques require complex or costly synthesis methods or can only be applied to a small class of materials. ${ }^{19,20}$ Furthermore, the resulting electrodes often lack the electrochemical and mechanical robustness necessary for long-term use or incorporation into flexible or stretchable devices. These disadvantages limit the opportunities for such materials to be scaled up to a commercial level.

Here we propose an alternative approach to improving ion mobility in supercapacitor electrodes: semi-interpenetrating polymer networks (sIPNs) of pseudocapacitive electrically conducting polymer in a cross-linked ionically conducting polymer matrix. The ionically conducting polymer generates an ion reservoir throughout the electrode, enabling pseudocapacitive polymer even within the bulk of the material to be accessible for charge storage reactions. This strategy increases the material utilization efficiency of the electrically conducting polymer without employing complex synthesis methods or sacrificing mechanical stability. Moreover, the flexible framework of the ionically conductive matrix can accommodate the volumetric changes associated with ion intercalation/de-intercalation in the pseudocapacitive polymer, minimizing mechanical stress in the electrode and yielding excellent cycling stability.

We demonstrate this interpenetrating network concept using poly(3-4,ethylenedioxythiophene) (PEDOT) and a poly(ethylene oxide) (PEO) based network (Figure 1a), a pair of polymers which has been studied extensively in the context of sIPNs for actuators, ${ }^{21-23}$ electrochromic devices, ${ }^{24,25}$ and tactile sensors ${ }^{26}$ but not yet explored for supercapacitor applications. PEDOT, the electrically 
conducting pseudocapacitive component of the sIPN, has the advantages of chemical and thermal stability, ${ }^{24}$ biocompatibility, ${ }^{27}$ and a competitive theoretical specific capacitance of $210 \mathrm{~F} / \mathrm{g} .{ }^{13}$ PEDOT also possesses high conductivity (up to $4500 \mathrm{~S} / \mathrm{cm}$ ) ${ }^{28,29}$ and can be operated under a larger voltage window than most other conducting polymers. ${ }^{30}$ The other portion of the sIPN, a crosslinked PEO-based network, achieves high ionic conductivity from its ethylene oxide groups, which can coordinate metal cations from the electrolyte ${ }^{21,31}$ and enhance the polarity of the material, improving its ability to swell with aqueous electrolyte. ${ }^{24}$ This swelling is also facilitated by dangling chains within the matrix, which create free space to further improve ion mobility. Our sIPN films (Figure 1b, c) are fabricated using a simple, two-step synthesis and demonstrate dramatically improved specific capacitance, cycling stability, and mechanical properties relative to electrodes made from conventional neat PEDOT.

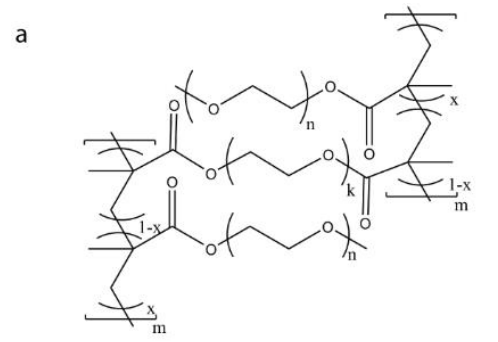

PEO-Based Network

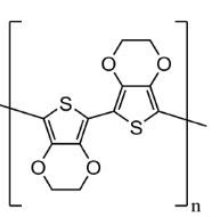

PEDOT
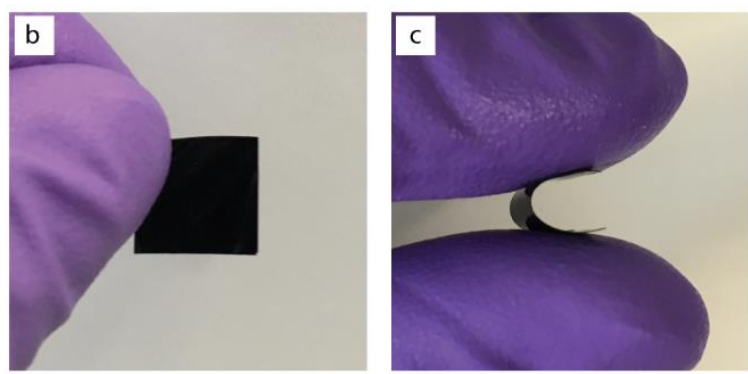

d

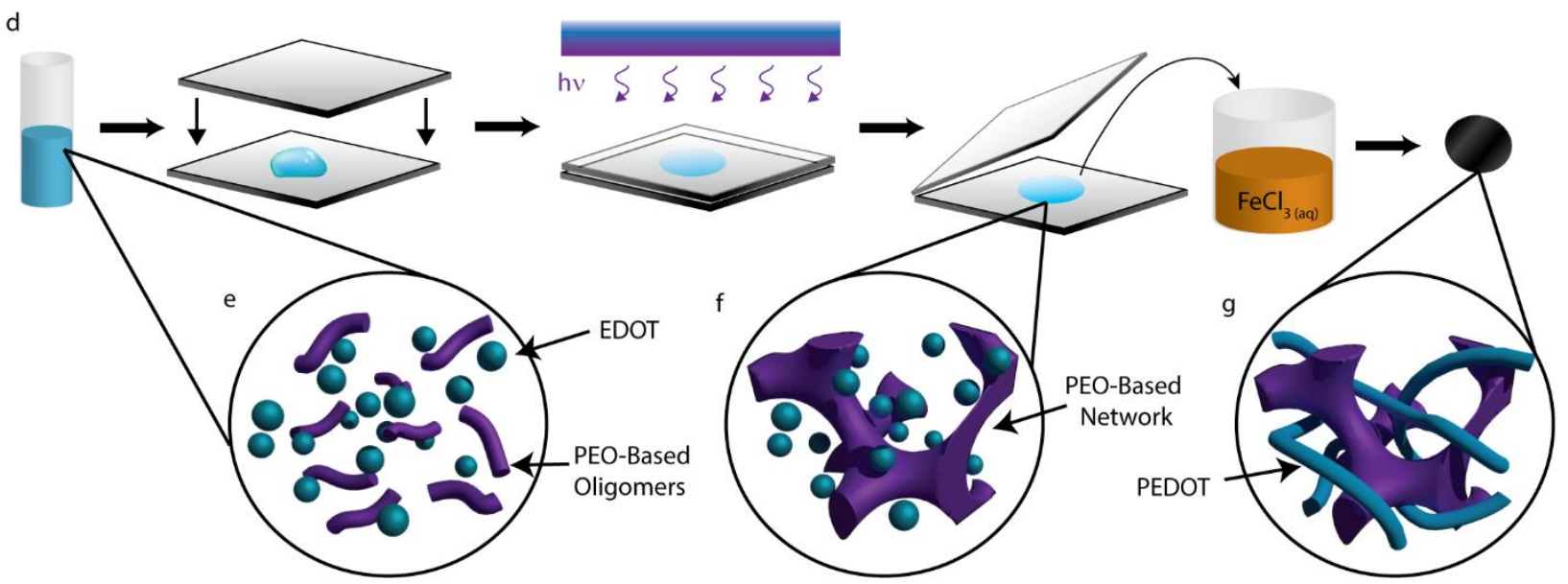

Figure 1. Preparation of sIPN films. (a) Chemical structures of the PEO-based network and PEDOT. (b, c) Photographs of the final sIPN films in flat and bent configurations, respectively. 
(d) Schematic of the sIPN synthesis procedure. (e-g) Illustration of the polymerization process. Step (e) illustrates the mixture of precursors, (f) shows the PEO matrix impregnated with EDOT, and $(\mathrm{g})$ is the final semi-interpenetrating network.

The sIPNs were synthesized according to the process in Figure 1d-g (see the Experimental Section in the Supporting Information). The PEO matrix was formed via free radical copolymerization of poly(ethylene glycol) methyl ether methacrylate (PEGM) and poly(ethylene glycol) dimethacrylate (PEGDM), using benzoin methyl ether (BME) as a photo-radical initiator. The PEGM:PEGDM ratio of 3:1 used here has been demonstrated in previous work to provide optimal ionic mobility; ${ }^{22}$ it allows for the maximum number of dangling chains within the matrix (provided by the PEGM) while maintaining the mechanical integrity enabled by cross-linking of the PEGDM. These dangling chains provide free volume to facilitate swelling of the gel with electrolyte - indeed, the area of these films increases by approximately $80 \%$ when wet. Raman spectroscopy of the films (Figure 2a) confirms the synthesis of a PEO-based material. ${ }^{32,33}$

To interpenetrate PEDOT within the films, EDOT was incorporated into the initial PEGM/PEGDM reagent mixture; crosslinking of the PEO network thus yielded EDOTimpregnated PEO gels. This EDOT was subsequently polymerized via chemical oxidative polymerization by immersing the films in $\mathrm{FeCl}_{3}$ solution, with $\mathrm{FeCl}_{3}$ serving as both oxidant and dopant. Given that EDOT is insoluble in aqueous solution, water was chosen as the solvent for the $\mathrm{FeCl}_{3}$ to minimize the migration of EDOT from the PEO gel.

The Raman spectrum of the sIPN film is overlaid with that of neat PEDOT in Figure $2 b$ to demonstrate the successful synthesis of PEDOT. ${ }^{34,35}$ In particular, the peak at $1425 \mathrm{~cm}^{-1}$ from symmetric $\mathrm{C}_{\alpha}=\mathrm{C}_{\beta}(-\mathrm{O})$ stretching indicates the presence of a high degree of conjugation in the PEDOT. ${ }^{36}$ We can further characterize the formation of PEDOT by quantifying the electrical 
conductivity of the film surface, which we measure to be $156 \mathrm{~S} / \mathrm{cm}$ using a four-point probe. This conductivity is of the same order as many other PEDOT-based supercapacitor materials from the literature, despite the addition of the electrically insulating PEO matrix. ${ }^{29,37}$ It should be cautioned, however, that these Raman spectroscopy and four-point probe measurements have only characterized the PEDOT at the surface of the sIPN. In order to ensure successful supercapacitor performance we must confirm the continuity of the PEDOT phase throughout the entire depth of the PEO matrix.
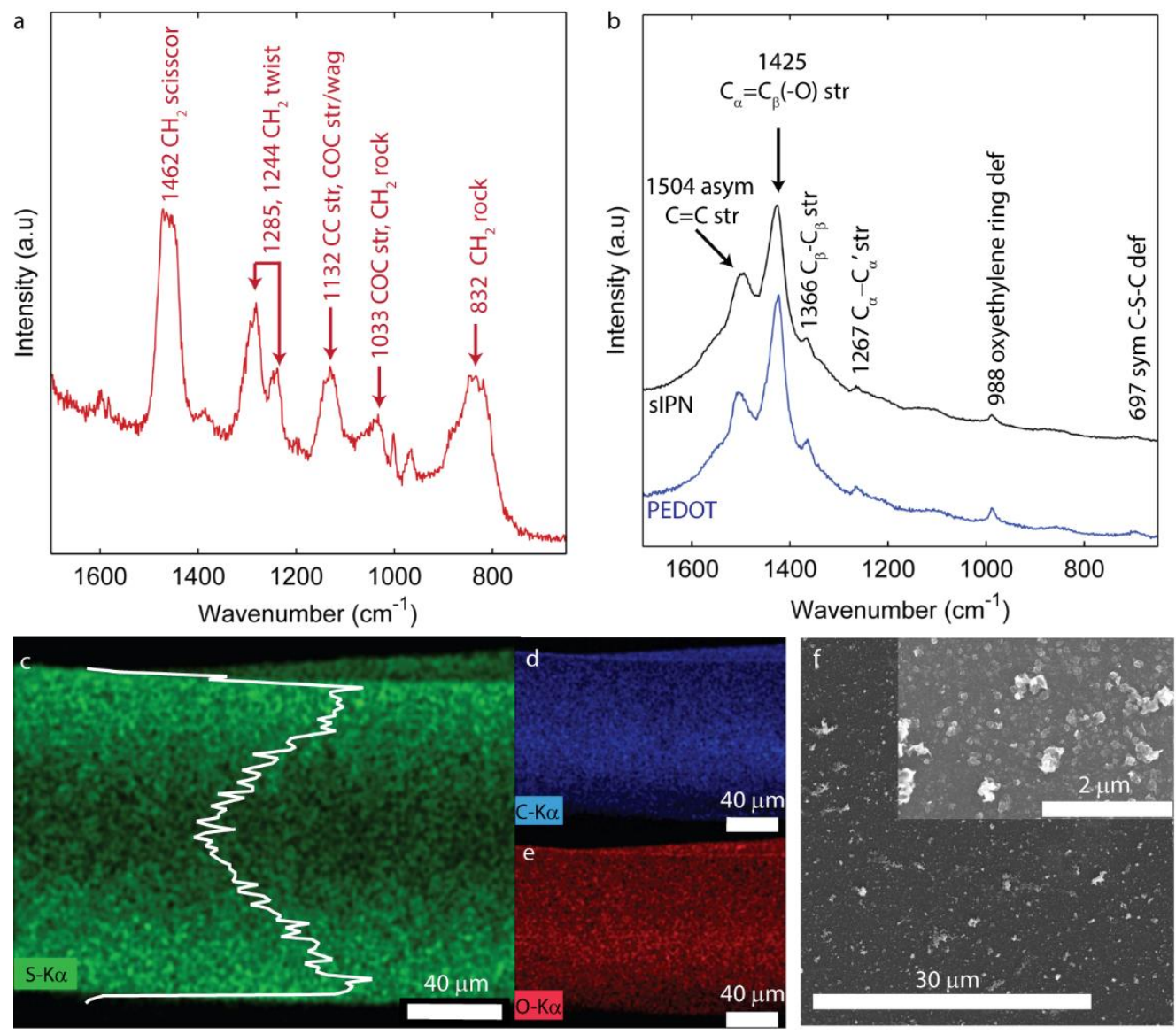

Figure 2. Characterization of the sIPN films. Raman spectra of (a) a PEO-based matrix (with no EDOT impregnated) and (b) a sIPN film, compared with neat PEDOT powder. (c-e) EDX elemental mapping of the sIPN film cross-section: (c) sulfur, overlaid with a semiquantitative plot of signal intensity, (d) carbon, and (e) oxygen. (f) SEM images of the sIPN surface. 
We probe the distribution of PEDOT throughout $130 \mu \mathrm{m}$ depth of the sIPN using energydispersive X-ray spectroscopy (EDX) mapping of the film cross-section: sulfur, which is present in PEDOT but not PEO, can be used to detect the distribution of PEDOT in the film. This analysis (Figure 2c) confirms the presence of PEDOT throughout the entire film, although we do observe decreased PEDOT content in the center of the film. Correspondingly, we observe increased carbon and oxygen signal in the film center (Figure 2d, e). The accumulation of PEDOT on the film surface is also apparent in scanning electron microscope (SEM) images (Figure 2f), in which we observe a relatively flat morphology with submicron PEDOT clusters on the surface.

We have thus demonstrated a two-step method to fabricate the sIPN electrodes, successfully achieving interconnectivity between the PEDOT and PEO-based matrix. In addition to its simplicity, the sIPN synthesis process is also highly tunable. The final PEDOT concentration in the films can be easily controlled, enabling a systematic optimization of the material's electrochemical and mechanical properties. For details of this process, which yielded an optimal PEDOT concentration of 61 wt. \%, see the Supporting Information (Figure S2 and Figure S3). Given the simplicity and controllability of this synthesis procedure, the methods used here for PEDOT and PEO could easily be transferred to a variety of other polymers for the development of additional interpenetrated supercapacitor materials.

Data from galvanostatic charge-discharge and cyclic voltammetry (CV) tests (Figure $3 \mathrm{a}$ and Figure $3 b$, respectively) demonstrate that the sIPNs exhibit a high specific capacitance of $182 \mathrm{~F} / \mathrm{g}$ at a charging rate of $1 \mathrm{~A} / \mathrm{g}(158 \mathrm{~F} / \mathrm{g}$ at $5 \mathrm{mV} / \mathrm{s})$. This is among the highest reported capacitance values for a PEDOT-based pure-polymer supercapacitor electrode (Table S1). ${ }^{29,38-41}$ The capacitance of the sIPN is especially impressive given its low surface area, inferred from its nitrogen adsorption isotherm, which indicates that the surface is nonporous (Figure S4). ${ }^{42}$ 

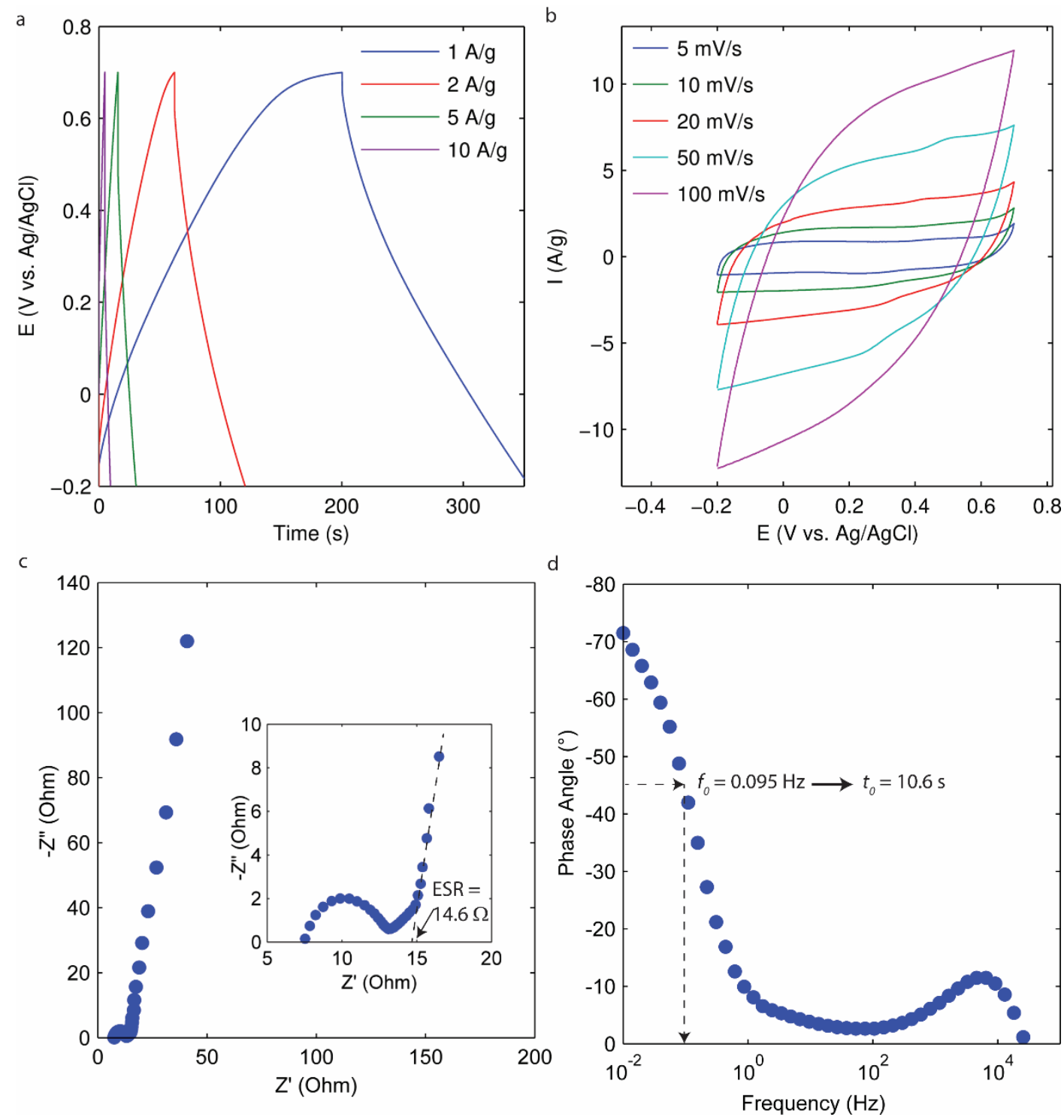

Figure 3. Electrochemical characterization of sIPN electrodes. (a) Galvanostatic charge-discharge data at rates of 1-10 A/g. (b) Cyclic voltammograms at scan rates ranging from $5 \mathrm{mV} / \mathrm{s}$ to 100 $\mathrm{mV} / \mathrm{s}$. (c) Nyquist plot, with the inset showing greater magnification of the high frequency region. (d) Bode plot showing impedance phase angle as a function of frequency.

The ionic/electronic resistances, charge transfer properties, and capacitive behavior of the sIPNs can be probed further using electrochemical impedance spectroscopy (EIS). The Nyquist plot in Figure 3c shows that the sIPN electrodes exhibit an equivalent series resistance (ESR) value of 
14.6 $\Omega$, obtained by extrapolating the vertical portion of the plot to the $\mathrm{x}$-axis. ${ }^{43}$ This ESR is only slightly higher than that of neat PEDOT samples despite the presence of approximately $40 \mathrm{wt} . \%$ PEO (Figure S5), indicating good continuity of the PEDOT phase throughout the electrode. In the low frequency region, the vertical slope of the plot indicates nearly ideal capacitive behavior in the electrode. This is represented more quantitatively by the y-intercept of the Bode plot (Figure 3d); the phase angle approaches $72^{\circ}$ at low frequencies, which is close to the $90^{\circ}$ angle of an ideal capacitor. The Bode plot can also provide insight into the rate capability of the electrodes. The frequency value at a phase angle of $-45^{\circ}\left(f_{0}\right)$ gives the dielectric relaxation time constant $\left(\tau_{0}\right)$ of the system, the minimum charge/discharge time at which the electrode can be operated with at least $50 \%$ efficiency. ${ }^{44,45}$ For the sIPN electrode, $f_{0}$ is $0.095 \mathrm{~Hz}$, corresponding to a $\tau_{0}$ of $10.6 \mathrm{~s}$. This value of $\tau_{0}$, which is comparable to the time constants for many other polymer-based supercapacitor materials, ${ }^{46,47}$ is most likely limited by the kinetics of ion diffusion within the PEO matrix as well as the charge transfer resistance of the pseudocapacitive charge storage processes.

In order to benchmark the sIPN performance against conventional materials, we synthesized a neat PEDOT powder via chemical oxidative polymerization, choosing reaction conditions which aligned as closely as possible with those of the PEDOT in the sIPNs, including consistent reaction time and oxidant concentration. This neat PEDOT provides a useful performance benchmark, although one has to take into account differences in surface area as well as the presence of binder and conductive additive required to fabricate the powder-based electrodes. As illustrated by the data in Figure $4 \mathrm{a}$ and Figure $4 \mathrm{~b}$, the sIPN electrodes exhibit up to $73 \%$ increased specific capacitance relative to the neat PEDOT value of $105 \mathrm{~F} / \mathrm{g}$ at $1 \mathrm{~A} / \mathrm{g}(91 \mathrm{~F} / \mathrm{g}$ at $5 \mathrm{mV} / \mathrm{s})$. Moreover, the sIPNs maintain higher specific capacitance values than the neat PEDOT even at high charging/discharging rates (Figure 4c). 

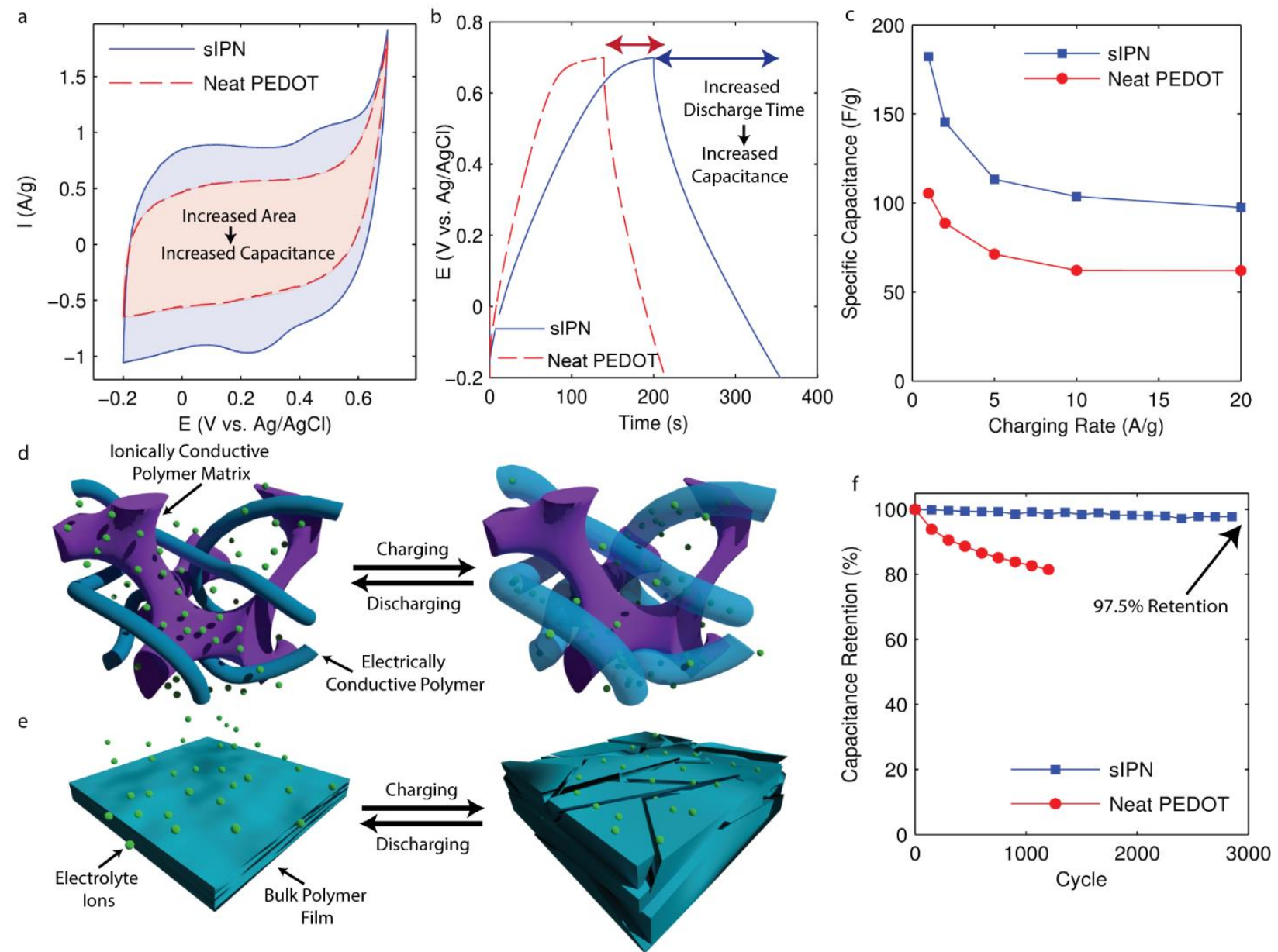

Figure 4. Performance advantages of the sIPN relative to neat PEDOT. (a) Cyclic voltammetry curves at $5 \mathrm{mV} / \mathrm{s}$. (b) Charge-discharge curves at $1 \mathrm{~A} / \mathrm{g}$. (c) Rate capability for charging rates up to $20 \mathrm{~A} / \mathrm{g}$. (d, e) Morphological origin of the specific capacitance and cycling stability trends of (d) the sIPN, where the crosslinked PEO matrix provides a reservoir of electrolyte ions and locally constrains swelling of the PEDOT during cycling, vs. (e) a bulk polymer film, where ion accessibility is limited to the electrode surface and swelling-induced strain can yield cracking and failure of the material. (f) Cycling stability, measured as retention of capacitance after repeated cycling at $10 \mathrm{~A} / \mathrm{g}$.

These improvements in specific capacitance emerge from the morphology of our sIPN material, illustrated schematically in Figure 4d. The ionically conductive PEO matrix acts as an ion reservoir 
surrounding the PEDOT, greatly reducing ion diffusion distances throughout the electrode relative to conventional structures. This enables PEDOT even within the bulk of the electrodes to participate in charge storage, as demonstrated by the fact that the specific capacitance of our sIPNs remains relatively constant when increasing the film thickness from $50 \mu \mathrm{m}$ to $130 \mu \mathrm{m}$ (Figure S6). Fabrication of electrodes which can maintain their specific capacitance at these relatively high thicknesses is much more difficult for conventional polymer films (Figure 4e), where poor ionic conductivity limits the access of electrolyte ions to a few tens of nanometers from the electrode surface. ${ }^{89}$ In fact, our high-performance $130 \mu \mathrm{m}$ films are one to two orders of magnitude thicker than many polymer-based electrodes reported in the literature. ${ }^{44,48-51}$

Our material morphology also greatly enhances electrode cycling stability, another crucial performance metric which presents particular challenges for polymer-based materials. Many polymer-based supercapacitor electrodes suffer from poor long-term capacitance retention due to the volumetric changes caused by repeated ion intercalation/de-intercalation. ${ }^{52,53}$ The resulting mechanical stress on the material can lead to issues such as delamination, collapse of ion flow channels, or disordering/breakage of polymer chains. ${ }^{54}$ This process is schematically illustrated for conventional bulk polymer morphologies in Figure 4e. Indeed, our neat PEDOT control sample showed only $82 \%$ capacitance retention after 1,200 cycles; other PEDOT-based materials in the literature have shown even poorer cycling stability. ${ }^{55-57}$ In contrast, the sIPN structure lends itself to excellent stability, retaining $97.5 \%$ of its initial specific capacitance after 3,000 cycles (Figure 4f). We hypothesize that the flexible, crosslinked PEO network in the sIPN acts as a mechanical buffer to accommodate volumetric changes upon cycling and thus suppress mechanical stress damage within the electrode (Figure 4d). Improved stability through similar mechanical buffering effects has been observed for a variety of composite polymer electrodes, with structures such as 
carbon nanotube networks, ${ }^{58}$ hydrogels,${ }^{59}$ or graphene oxide sheets ${ }^{60}$ providing flexible or open structures to minimize the negative effects of repeated swelling/shrinking. The sIPN electrode also maintained effectively $100 \%$ Coulombic efficiency over the 3,000 cycles (Figure S7), characteristic of highly reversible (pseudo)capacitive processes and a lack of parasitic side reactions.

In addition to improved specific capacitance and cycling stability, the sIPN electrodes surpass our neat PEDOT samples in their mechanical properties as well. The sIPNs are formed as freestanding films, eliminating the need for additional binders or substrates which can decrease specific capacitance by adding inactive weight to the electrode. Furthermore, the sIPN films are flexible, as pictured in Figure 1c and Figure S8a. The $130 \mu \mathrm{m}$ thick films can be bent to radii of curvature below $200 \mu \mathrm{m}$ without breaking. Even after 1,000 cycles of bending/unbending, the CV profile of the electrode remains essentially unchanged (Figure S8b), retaining $99 \%$ of its initial capacitance. Further characterization of the sIPN mechanical properties can be found in Figure S9.

As a preliminary investigation of the potential applicability of these electrodes, a full solid-state supercapacitor device was fabricated using two identical pieces of the sIPN and a PEO-based gel as the electrolyte (Figure S10a). Based on cyclic voltammetry data (Figure S10b), the device capacitance reached $28.8 \mathrm{~F} / \mathrm{g}$ at a scan rate of $5 \mathrm{mV} / \mathrm{s}$, corresponding to an energy density of 3.2 $\mathrm{Wh} / \mathrm{kg}$ at a power density of $64.8 \mathrm{~W} / \mathrm{kg}$. Two devices in series proved sufficiently powerful to light a LED (Figure S10c). This proof-of-concept demonstration suggests the potential of sIPNbased electrodes to be utilized for novel applications such as wearable or implantable electronics.

We have demonstrated that interpenetrating ionically and electrically conducting polymers is a successful strategy to improve the performance of supercapacitor electrodes. The semiinterpenetrating polymer network (sIPN) structure optimizes the accessibility of the entire 
pseudocapacitive polymer to electrolyte ions, resulting in specific capacitance over $70 \%$ greater than that of neat PEDOT powder. Furthermore, the robust mechanical structure of the PEO phase confines volumetric changes in the PEDOT upon cycling, minimizing mechanical stress on the electrode to yield drastically improved cycling stability. Finally, the resulting tough and flexible materials (bendable to $<200 \mu \mathrm{m}$ radius of curvature) are promising for wearable electronics and other flexible technologies. This sIPN approach has the potential to be generalized as a framework to improve the performance of a myriad of polymer-based energy storage materials.

\section{ASSOCIATED CONTENT}

Supporting Information. Experimental section; effect of PEDOT loading on sIPN performance; nitrogen adsorption isotherm; electrochemical impedance data for neat PEDOT control sample; effect of film thickness on specific capacitance; coulombic efficiency over longterm cycling; sIPN compressive stress-strain curve; characterization of solid-state supercapacitor.

\section{AUTHOR INFORMATION}

\section{Corresponding Author}

*E-mail: sks46@cam.ac.uk

\section{Author Contributions}

${ }^{\dagger}$ Kara D. Fong and Tiesheng Wang contributed equally to this work.

\section{Notes}

The authors declare no competing financial interest.

\section{ACKNOWLEDGMENT}


This work was funded by the European Research Council (ERC) grant to S.K.S., EMATTER (\# 280078). K.D.F. acknowledges support from the Winston Churchill Foundation of the United

States. T.W. thanks the China Scholarship Council (CSC) for funding and the Engineering and Physical Sciences Research Council of the UK (EPSRC) Centre for Doctoral Training in Sensor Technologies and Applications (grant number: EP/L015889/1) for support.

\section{REFERENCES}

(1) Miller, J. R.; Burke, A. F. Electrochemical Capacitors: Challenges and Opportunities for Real-World Applications. Electrochem. Soc. Interface 2008, 17, 53-57.

(2) Simon, P.; Gogotsi, Y. Charge Storage Mechanism in Nanoporous Carbons and Its Consequence for Electrical Double Layer Capacitors. Philos. Trans. R. Soc. A Math. Phys. Eng. Sci. 2010, 368, 3457-3467.

(3) Miller, J. M.; Everett, M. Ultra-Capacitor Augmentation of the Vehicle Electrical System to Reset Its Power Budget. Power Electron. Transp. 2004, 19-26.

(4) Wang, L.; Wu, Q.; Zhang, Z.; Zhang, Y.; Pan, J.; Li, Y.; Zhao, Y.; Zhang, L.; Cheng, X.; Peng, H. Elastic and Wearable Ring-Type Supercapacitors. J. Mater. Chem. A 2016, 4, $3217-3222$.

(5) Choi, C.; Kim, S. H.; Sim, H. J.; Lee, J. A.; Choi, A. Y.; Kim, Y. T.; Lepró, X.; Spinks, G. M.; Baughman, R. H.; Kim, S. J. Stretchable, Weavable Coiled Carbon Nanotube/MnO $2 /$ Polymer Fiber Solid-State Supercapacitors. Sci. Rep. 2015, 5, 9387.

(6) Wang, T.; Farajollahi, M.; Choi, Y. S.; Lin, I.-T.; Marshall, J. E.; Thompson, N. M.; KarNarayan, S.; Madden, J. D. W.; Smoukov, S. K. Electroactive Polymers for Sensing. 
Interface Focus 2016, 6, 20160026.

(7) Yang, M.; Xia, H. Exploration and Progress of High-Energy Supercapacitors and Related Electrode Materials. Sci. China Technol. Sci. 2015, 58, 1851-1863.

(8) Horng, Y.-Y.; Lu, Y.-C.; Hsu, Y.-K.; Chen, C.-C.; Chen, L.-C.; Chen, K.-H. Flexible Supercapacitor Based on Polyaniline Nanowires/carbon Cloth with Both High Gravimetric and Area-Normalized Capacitance. J. Power Sources 2010, 195, 4418-4422.

(9) Simon, P.; Gogotsi, Y. Materials for Electrochemical Capacitors. Nat. Mater. 2008, 7, 845854.

(10) Chabi, S.; Peng, C.; Hu, D.; Zhu, Y. Ideal Three-Dimensional Electrode Structures for Electrochemical Energy Storage. Adv. Mater. 2014, 26, 2440-2445.

(11) Zhou, C.; Zhang, Y.; Li, Y.; Liu, J. Construction of High-Capacitance 3D CoO@Polypyrrole Nanowire Array Electrode for Aqueous Asymmetric Supercapacitor. Nano Lett. 2013, 13, 2078-2085.

(12) Jabeen, N.; Xia, Q.; Yang, M.; Xia, H. Unique Core-Shell Nanorod Arrays with Polyaniline Deposited into Mesoporous $\mathrm{NiCo}_{2} \mathrm{O}_{4}$ Support for High-Performance Supercapacitor Electrodes. ACS Appl. Mater. Interfaces 2016, 8, 6093-6100.

(13) Lota, K.; Khomenko, V.; Frackowiak, E. Capacitance Properties of poly(3,4Ethylenedioxythiophene)/carbon Nanotubes Composites. J. Phys. Chem. Solids 2004, 65, 295-301.

(14) Guo, F. M.; Xu, R. Q.; Cui, X.; Zhang, L.; Wang, K. L.; Yao, Y. W.; Wei, J. Q. High Performance of Stretchable Carbon Nanotube-polypyrrole Fiber Supercapacitors under 
Dynamic Deformation and Temperature Variation. J. Mater. Chem. A 2016, 4, 9311-9318.

(15) Yan, X.; Tai, Z.; Chen, J.; Xue, Q. Fabrication of Carbon Nanofiber-polyaniline Composite Flexible Paper for Supercapacitor. Nanoscale 2011, 3, 212-216.

(16) Han, Y.; Shen, M.; Wu, Y.; Zhu, J.; Ding, B.; Tong, H.; Zhang, X. Preparation and Electrochemical Performances of PEDOT/sulfonic Acid-Functionalized Graphene Composite Hydrogel. Synth. Met. 2013, 172, 21-27.

(17) Zhou, H.; Yao, W.; Li, G.; Wang, J.; Lu, Y. Graphene/poly(3,4-Ethylenedioxythiophene) Hydrogel with Excellent Mechanical Performance and High Conductivity. Carbon 2013, $59,495-502$.

(18) Guo, H.; He, W.; Lu, Y.; Zhang, X. Self-Crosslinked Polyaniline Hydrogel Electrodes for Electrochemical Energy Storage. Carbon 2015, 92, 133-141.

(19) Zhu, C.; Kopold, P.; Van Aken, P. A.; Maier, J.; Yu, Y. High Power-High Energy Sodium Battery Based on Threefold Interpenetrating Network. Adv. Mater. 2016, 28, 2409-2416.

(20) Nyström, G.; Marais, A.; Karabulut, E.; Wågberg, L.; Cui, Y.; Hamedi, M. M. SelfAssembled Three-Dimensional and Compressible Interdigitated Thin-Film Supercapacitors and Batteries. Nat. Commun. 2015, 6, 7259.

(21) Khaldi, A.; Plesse, C.; Vidal, F.; Smoukov, S. K. Smarter Actuator Design with Complementary and Synergetic Functions. Adv. Mater. 2015, 27, 4418-4422.

(22) Festin, N.; Maziz, A.; Plesse, C.; Teyssié, D.; Chevrot, C.; Vidal, F. Robust Solid Polymer Electrolyte for Conducting IPN Actuators. Smart Mater. Struct. 2013, 22, 104005. 
(23) Plesse, C.; Vidal, F.; Randriamahazaka, H.; Teyssié, D.; Chevrot, C. Synthesis and Characterization of Conducting Interpenetrating Polymer Networks for New Actuators. Polymer (Guildf). 2005, 46, 7771-7778.

(24) Vidal, F.; Plesse, C.; Aubert, P. H.; Beouch, L.; Tran-Van, F.; Palaprat, G.; Verge, P.; Yammine, P.; Citerin, J.; Kheddar, A.; et al. Poly(3,4-Ethylenedioxythiophene)-Containing Semi-Interpenetrating Polymer Networks: A Versatile Concept for the Design of Optical or Mechanical Electroactive Devices. Polym. Int. 2010, 59, 313-320.

(25) Tran-Van, F.; Beouch, L.; Vidal, F.; Yammine, P.; Teyssié, D.; Chevrot, C. Self-Supported Semi-Interpenetrating Polymer Networks for New Design of Electrochromic Devices. Electrochim. Acta 2008, 53, 4336-4343.

(26) Festin, N.; Plesse, C.; Chevrot, C.; Teyssié, D.; Josselin, L.; Pirim, P.; Vidal, F. Actuation and Sensing Properties of Electroactive Polymer Whiskers. Procedia Comput. Sci. 2011, 7 , S4-S7.

(27) Balint, R.; Cassidy, N. J.; Cartmell, S. H. Conductive Polymers: Towards a Smart Biomaterial for Tissue Engineering. Acta Biomater. 2014, 10, 2341-2353.

(28) Kim, J. Y.; Kwon, M. H.; Min, Y. K.; Kwon, S.; Ihm, D. W. Self-Assembly and Crystalline Growth of poly(3,4-Ethylenedioxythiophene) Nanofilms. Adv. Mater. 2007, 19, 35013506.

(29) D’Arcy, J. M.; El-Kady, M. F.; Khine, P. P.; Zhang, L.; Lee, S. H.; Davis, N. R.; Liu, D. S.; Yeung, M. T.; Kim, S. Y.; Turner, C. L.; et al. Vapor-Phase Polymerization of Nanofibrillar Poly(3,4-Ethylenedioxythiophene) for Supercapacitors. ACS Nano 2014, 8, 1500-1510. 
(30) Shown, I.; Ganguly, A.; Chen, L.-C.; Chen, K.-H. Conducting Polymer-Based Flexible Supercapacitor. Energy Sci. Eng. 2015, 3, 2-26.

(31) Gauthier, C.; Plesse, C.; Vidal, F.; Pelletier, J. M.; Chevrot, C.; Teyssié, D. Polybutadiene/poly(ethylene Oxide) Based IPNs, Part II: Mechanical Modelling and $\mathrm{LiClO}_{4}$ Loading as Tools for IPN Morphology Investigation. Polymer (Guildf). 2007, 48, 7476-7483.

(32) Westover, A. S.; Shabab, F. N.; Tian, J. W.; Bernath, S.; Oakes, L.; Erwin, W. R.; Carter, R.; Bardhan, R.; Pint, C. L. Stretching Ion Conducting Polymer Electrolytes: In-Situ Correlation of Mechanical, Ionic Transport, and Optical Properties. J. Electrochem. Soc. 2014, 161, E112-E117.

(33) Kotzianova, A.; Rebicek, J.; Zidek, O.; Pokorny, M.; Hrbac, J.; Velebny, V. Raman Spectroscopy Based Method for the Evaluation of Compositional Consistency of Nanofibrous Layers. Anal. Methods 2015, 7, 9900-9905.

(34) Wang, T.; Farajollahi, M.; Henke, S.; Zhu, T.; Bajpe, S. R.; Sun, S.; Barnard, J. S.; Lee, J. S.; Madden, J. D. W.; Cheetham, A. K.; et al. Functional Conductive Nanomaterials via Polymerisation in Nano-Channels: PEDOT in a MOF. Mater. Horiz. 2016, 310, 1166-1170.

(35) Winther-Jensen, B.; West, K. Vapor-Phase Polymerization of 3,4-Ethylenedioxythiophene: A Route to Highly Conducting Polymer Surface Layers. Macromolecules 2004, 37, 45384543.

(36) Wu, D.; Zhang, J.; Dong, W.; Chen, H.; Huang, X.; Sun, B.; Chen, L. Temperature Dependent Conductivity of Vapor-Phase Polymerized PEDOT Films. Synth. Met. 2013, 
$176,86-91$.

(37) Laforgue, A. All-Textile Flexible Supercapacitors Using Electrospun poly(3,4Ethylenedioxythiophene) Nanofibers. J. Power Sources 2011, 196, 559-564.

(38) Li, W.; Chen, J.; Zhao, J.; Zhang, J.; Zhu, J. Application of Ultrasonic Irradiation in Preparing Conducting Polymer as Active Materials for Supercapacitor. Mater. Lett. 2004, $59,800-803$.

(39) Zhao, Q.; Jamal, R.; Zhang, L.; Wang, M.; Abdiryim, T. The Structure and Properties of PEDOT Synthesized by Template-Free Solution Method. Nanoscale Res. Lett. 2014, 9, 557.

(40) Tong, L.; Skorenko, K. H.; Faucett, A. C.; Boyer, S. M.; Liu, J.; Mativetsky, J. M.; Bernier, W. E.; Jones, W. E. Vapor-Phase Polymerization of poly(3,4-Ethylenedioxythiophene) (PEDOT) on Commercial Carbon Coated Aluminum Foil as Enhanced Electrodes for Supercapacitors. J. Power Sources 2015, 297, 195-201.

(41) Bai, X.; Hu, X.; Zhou, S.; Yan, J.; Sun, C.; Chen, P.; Li, L. 3D Flowerlike poly(3,4Ethylenedioxythiophene) for High Electrochemical Capacitive Energy Storage. Electrochim. Acta 2013, 106, 219-225.

(42) Sing, K. S. W. Reporting Physisorption Data for Gas/solid Systems with Special Reference to the Determination of Surface Area and Porosity (Recommendations 1984). Pure Appl. Chem. 1985, 57, 603-619.

(43) Kim, T.; Jung, G.; Yoo, S.; Suh, K. S.; Ruoff, R. S. Activated Graphene-Based Carbons as Supercapacitor Electrodes with Macro- and Mesopores. ACS Nano 2013, 7, 6899-6905.

(44) Choi, B. G.; Hong, J.; Hong, W. H.; Hammond, P. T.; Park, H. Facilitated Ion Transport in 
All-Solid-State Flexible Supercapacitors. ACS Nano 2011, 5, 7205-7213.

(45) Feng, H.; Zheng, M.; Dong, H.; Xiao, Y.; Hu, H.; Sun, Z.; Long, C.; Cai, Y.; Zhao, X.; Zhang, H.; et al. Three-Dimensional Honeycomb-like Hierarchically Structured Carbon for High-Performance Supercapacitors Derived from High-Ash-Content Sewage Sludge. J. Mater. Chem. A 2015, 3, 15225-15234.

(46) Gottam, R.; Srinivasan, P. One-Step Oxidation of Aniline by Peroxotitanium Acid to Polyaniline-Titanium Dioxide: A Highly Stable Electrode for a Supercapacitor. J. Appl. Polym. Sci. 2015, 132, 41711.

(47) Zhang, K.; Hu, H.; Yao, W.; Ye, C. Flexible and All-Solid-State Supercapacitors with LongTime Stability Constructed on PET/Au/polyaniline Hybrid Electrodes. J. Mater. Chem. A 2015, 3, 617-623.

(48) Kaempgen, M.; Chan, C. K.; Ma, J.; Cui, Y.; Gruner, G. Printable Thin Film Supercapacitors Using Single-Walled Carbon Nanotubes. Nano Lett. 2009, 9, 1872-1876.

(49) Choi, K. S.; Liu, F.; Choi, J. S.; Seo, T. S. Fabrication of Free-Standing Multilayered Graphene and Poly(3,4-Ethylenedioxythiophene) Composite Films with Enhanced Conductive and Mechanical Properties. Langmuir 2010, 26, 12902-12908.

(50) Jo, K.; Lee, T.; Choi, H. J.; Park, J. H.; Lee, D. J.; Lee, D. W.; Kim, B.-S. Stable Aqueous Dispersion of Reduced Graphene Nanosheets via Non-Covalent Functionalization with Conducting Polymers and Application in Transparent Electrodes. Langmuir 2011, 27, 2014-2018.

(51) Liu, Y.; Weng, B.; Razal, J. M.; Xu, Q.; Zhao, C.; Hou, Y.; Seyedin, S.; Jalili, R.; Wallace, 
G. G.; Chen, J. High-Performance Flexible All-Solid-State Supercapacitor from Large FreeStanding Graphene-PEDOT/PSS Films. Sci. Rep. 2015, 5, 17045.

(52) Frackowiak, E.; Khomenko, V.; Jurewicz, K.; Lota, K.; Béguin, F. Supercapacitors Based on Conducting Polymers/nanotubes Composites. J. Power Sources 2006, 153, 413-418.

(53) Liu, T.; Finn, L.; Yu, M.; Wang, H.; Zhai, T.; Lu, X.; Tong, Y.; Li, Y. Polyaniline and Polypyrrole Pseudocapacitor Electrodes with Excellent Cycling Stability. Nano Lett. 2014, $14,2522-2527$.

(54) Bryan, A. M.; Santino, L. M.; Lu, Y.; Acharya, S.; D’Arcy, J. M. Conducting Polymers for Pseudocapacitive Energy Storage. Chem. Mater. 2016, 28, 5989-5998.

(55) Bai, X.; Hu, X.; Zhou, S.; Yan, J.; Sun, C.; Chen, P.; Li, L. In Situ Polymerization and Characterization of Grafted Poly (3,4-Ethylenedioxythiophene)/multiwalled Carbon Nanotubes Composite with High Electrochemical Performances. Electrochim. Acta 2013, $87,394-400$.

(56) Yang, Y.; Zhang, L.; Li, S.; Yang, W.; Xu, J.; Jiang, Y.; Wen, J. Electrochemical Performance of Conducting Polymer and Its Nanocomposites Prepared by Chemical Vapor Phase Polymerization Method. J. Mater. Sci. Mater. Electron. 2013, 24, 2245-2253.

(57) Lee, S.; Cho, M. S.; Lee, H.; Nam, J.-D.; Lee, Y. A Facile Synthetic Route for Well Defined Multilayer Films of Graphene and PEDOT via an Electrochemical Method. J. Mater. Chem. 2012, 22, 1899-1903.

(58) Chen, Y.; Du, L.; Yang, P.; Sun, P.; Yu, X.; Mai, W. Significantly Enhanced Robustness and Electrochemical Performance of Flexible Carbon Nanotube-Based Supercapacitors by 
Electrodepositing Polypyrrole. J. Power Sources 2015, 287, 68-74.

(59) Hao, G.-P.; Hippauf, F.; Oschatz, M.; Wisser, F. M.; Leifert, A.; Nickel, W.; MohamedNoriega, N.; Zheng, Z.; Kaskel, S. Stretchable and Semitransparent Conductive Hybrid Hydrogels for Flexible Supercapacitors. ACS Nano 2014, 8, 7138-7146.

(60) Salunkhe, R. R.; Hsu, S.-H.; Wu, K. C. W.; Yamauchi, Y. Large-Scale Synthesis of Reduced Graphene Oxides with Uniformly Coated Polyaniline for Supercapacitor Applications. ChemSusChem 2014, 7, 1551-1556. 